Final version published in Sociological Forum

https://doi-org /10.1111/socf.12686

\title{
How Cultural Environments Shape Online Sentiment Toward Social Movements: Place Character and Support for Feminism
}

\author{
William J. Scarborough ${ }^{1}$ and Allison Suppan Helmuth ${ }^{2}$
}

\begin{abstract}
This study explores the relationship between offline and online contexts in social movement discourse. We test whether local place character - the cultural reputations, values, and ideals associated with a particular location-predict support for feminism expressed on Twitter. Applying topic modeling to a unique corpus describing cities' cultural reputations, we identify five cultural attributes of U.S. cities that constitute their place character. Then, we use these measures to predict the percentage of tweets that are positive toward feminism in each location. The results indicate that while social media may connect people from geographically distant areas, online discourse is still heavily influenced by the local spaces where users reside. Cities with a place character of Revitalization have more positive Twitter sentiment toward feminism, while areas known as Local Hubs for shopping and leisure are more negative. These findings contribute to social movement scholarship by highlighting one way that offline and online contexts are connected. Far from transcending local geographies, social movement discourse taking place online remains shaped by the cultural environment where it originates.
\end{abstract}

Keywords: Social Movements, Feminism, Place Character, Culture, Twitter, Topic Modeling

The authors would like to thank Alison Dahl Crossley for their insightful comments on earlier versions of this manuscript. This research was supported by the Alice J. Dan Award for Research on Women and Gender at the University of Illinois at Chicago.

\footnotetext{
${ }^{1}$ Department of Sociology, University of North Texas, 1155 Union Circle, Denton, TX 76203; email: william.scarborough@unt.edu

${ }^{2}$ Department of Sociology, University of Illinois at Chicago, 1007 W. Harrison St., Chicago, IL 60607; e-mail: ahelmu2@uic.edu
} 
Place Character and Online Support for Feminism

\section{INTRODUCTION}

Research on the relationship between social movements and local context provides two accounts. On one hand, studies have found that discourse taking place on blogs and social media has transcended spatial constraints by allowing those with shared political interests and viewpoints to connect regardless of distance (Crossley and Nelson 2018; Earl and Kimport 2011; Taylor 2016). Other research, however, has found that local cultural conditions continue to have a large influence on support for social movements (Guenther 2010; Reger 2012). One reason for these different points of emphasis is that the former body of research has tended to focus on online settings, while the latter examines communities in offline, physical spaces. Yet, online and offline social dynamics do not occur in isolation from one another, as the users and creators of online content are always emplaced in specific offline, geographic contexts (Graham et al. 2012). Online engagement with social movements can shape opinions expressed within in-person settings, while individuals' experiences in offline environments may influence viewpoints made on social media (Earl 2015). Although often studied independently, online and offline settings are inherently connected by the individuals who simultaneously exist within both domains.

This study bridges research on offline and online social movement engagement to test whether local cultural environments across the U.S. shape levels of online support for feminism, a predominant social movement with a large social media presence (Crossley 2017). We conceptualize local culture through applying the concept of "place character" which describes the collection of cultural reputations, values, and community ideals associated with a particular location that emerge in popular discourse and shape residents' perceptions of themselves and social issues (Brown-Saracino 2017; Molotch et al. 2000; Paulsen 2004). Using new methods for the study of culture, we apply topic modeling to a corpus of online travel guide pages that 
describe the reputations of 133 cities across the U.S. and identify five primary themes constituting local place character. Then, we examine whether these cultural attributes are related to the percentage of tweets that are positive toward feminism as a measure of online support for this particular social movement.

Our results indicate that despite the capability for social media websites like Twitter to transcend space, local geographies are still deeply related to online feminist discourse. Areas with a place character of Revitalization (e.g. cultural and economic change following a period of economic decline) have higher levels of support for feminism on Twitter, while those known as regional shopping hubs have more negative sentiment toward feminism. These results shed new light on the relationship between physical space and online space in local feminist discourse, indicating that this social movement receives greater endorsement online in areas that are experiencing broader social change. More generally, findings from this study inform research on social movements by highlighting the dynamic relationship between online and offline settings. Far from transcending geographic space, online social movement discourse remains tied to the place character of the particular geographic areas in which users reside.

\section{SPATIAL CONTEXT AND SUPPORT FOR FEMINISM}

Scholars have devoted significant attention to studying how local conditions influence both the emergence and trajectories of social movements such as feminism (Guenther 2010; Hurwitz and Taylor 2012; Reger 2012). In general, research has found that local cultural conditions provide opportunities for social movements to emerge when collective identities make groups more perceptive of injustice and, therefore, motivate unified action (Polletta and Jasper 2001). Studies focusing on the feminist movement have provided significant evidence for this trend both historically (McCammon et al. 2007) and in more contemporary contexts (Reger 
2012). These works have found that individuals more readily identify with and support feminism during periods of broader social change (McCammon et al. 2007) and in more liberal locations such as college campuses (Reger 2012) and cities (Guenther 2010). Rather than identification with feminism reflecting purely personal endeavors, this research indicates that it is influenced by individuals' social and cultural environment.

Studying the contexts surrounding collective identification and support toward feminism, scholars have increasingly directed attention to the cultural attributes of the places where feminism is supported or where it meets resistance. As opposed to the economic or demographic characteristics of place, this body of research illustrates how cultural features, such as local reputations, traditions, and community identities, create the conditions that are more or less conducive to feminist organizing (Hurwitz and Taylor 2012). Guenther (2010), for example, compared two German cities that were similar in most demographic and economic indicators, but had very different local histories and cultural reputations. In one city, values and norms around personal independence fostered an environment where feminist values were supported and mainstreamed into local politics. In the other, socially conservative traditions presented challenges for local feminists who met resistance when pursuing agendas that contradicted essentialist notions of gender difference. According to Guenther, the varying levels of support for feminism in these cities stemmed from their differing place character - the collection of cultural reputations, values, and community identities that shape, in her study, how residents felt toward feminist ideals.

Guenther's application of place character to study communities' reception of feminism draws upon a longstanding body of urban culturalist scholarship that examines the role of local cultural characteristics in shaping the symbolic representations and reputations of cities (Borer 
2006) and their impact on individuals' daily lives and community outcomes (Molotch et al. 2000; Paulsen 2004). Brown-Saracino (2017: 15) defines place character as "the stories residents tell about their city to themselves," which "offer models for who and how to be in a place." Place character emerges in residents' descriptions of areas as well as through the billboards, fliers, and promotional materials that convey qualities defining both community values and the type of people who reside there (Molotch 2002; Paulsen 2004). From this perspective, local cultural reputations are not idle monikers for the places we live, but instead shape how residents perceive themselves, those in their community, and their attitudes toward public issues (Borer 2006). Scholars have found, for example, that place character can influence residents' expression of individual gender identity (Abelson 2019), their interactions with community groups (BrownSaracino 2017), and the design of public policies (Molotch et al. 2000).

Research on feminism in the U.S., while not explicitly invoking the concept of place character, nonetheless highlights the importance of these cultural features. Comparative work on U.S. universities has found that cultural conservatism on certain campuses poses major barriers to feminist groups seeking institutional support and engagement with student bodies (Crossley 2017; Reger 2012). In these settings, students may view feminism as antithetical to their universities' conservative values and traditions. Other research has found that feminist organizations and institutions are clustered within certain areas of the U.S., with some locations having a greater prevalence of women's centers, bookstores, and social groups that advance feminist causes and rally local support (Spain 2017). This research indicates that feminism receives more support in environments with a greater prevalence of cultural institutions that convey values of social equity and inclusion. 
Although there is substantial evidence that local cultural conditions predispose residents to embracing or rejecting feminism, this research has primarily focused on offline settings where people interact, communicate, and debate in physical spaces. In such contexts, the role of local place character may be highly salient, as offline communities exist within physically specific, socially constructed spaces that are imbued with cultural meanings, histories, and traditions. Online spaces such as social media, in contrast, may be less rooted to physically-specific environments. By allowing individuals to engage with others from across the globe, perceptions of social movements like feminism may be untethered from geographically emplaced communities. As a result, it is possible that viewpoints toward feminism expressed online are unrelated to the local place character where social media users generate content and reside.

\section{ONLINE FEMINISM: TRANSCENDING GEOGRAPHIES?}

The study of local place character and support for feminism has been primarily constrained to offline settings. However, online spaces provide opportunities for individuals to transcend geographical constraints which may limit expressions of support in their community. Highly visible campaigns such as \#MeToo and the Women's March have generated massive online followings where social media often serves as a venue for individuals to learn about, participate, and exchange viewpoints on these social movements (Crossley 2019). Indeed, recent research has shown that social media has facilitated a contemporary resurgence of feminism as users from across the world are able to connect, form collective identity, and express support regardless of the local conditions where they reside (Crossley 2017; Turley and Fisher 2018).

Although online settings provide the capability to transcend geographical constraints, place character and spatial context may nonetheless influence views expressed online. In particular, scholars have argued that place character shapes individuals' perceptions of 
themselves and their values (Brown-Saracino 2017; Molotch et al. 2000). From this perspective, it is possible that local place character may predispose social media users to certain views toward feminism that are then shared with a more global audience through social media websites like Twitter. An individual adhering to the conservative values of their home-town, for example, may be similarly resistant to feminism in discussions with neighbors as they are in online discussions with social media users across the country. Despite increased capabilities for global connection, sentiment toward social movements expressed online may nonetheless be influenced by the same dynamics affecting views expressed offline. Indeed, Earl (2014) suggests that although social media provides many new opportunities for social movement engagement, it nonetheless encounters many of the same challenges of garnering support faced by in-person organizing. This pattern may hold true in the case of local place character - just as it shapes perceptions of feminism in offline communities, it may similarly impact discourse taking place online.

\section{CURRENT STUDY}

In this study, we test whether local place character is related to feminist sentiment expressed online. Drawing from a unique corpus describing the cultural environments of U.S. cities, we examine how areas' cultural reputations relate to sentiment toward feminism occurring on Twitter as a social media platform that has played a large role in the growth of online feminist activism. ${ }^{3}$ By examining the relationship between local geographical context and online sentiment toward feminism, we deepen our understanding of the factors contributing to public

\footnotetext{
${ }^{3}$ Although research shows that Facebook is useful in facilitating feminist groups and online communities, these same studies argue that Twitter has a broader reach and better captures public discourse (Crossley 2017, 2019). Twitter allows users to follow hashtags unassociated with a specific place. It is also more common on Twitter than Facebook for users to follow individuals they've never met and who reside in a distant location (Lim and Datta 2012). Therefore, our use of Twitter focuses on a form of social media tied closely to public discourse and with greater ability to transcend geographical constraints.
} 
opinion toward social movements in online spaces. In addition, this study advances our knowledge on the relationship between physical and online spaces. Local culture and place character have been found to play a large role in perceptions of feminism within offline communities, but does this relationship also exist in online spaces? Does discourse on social media transcend the geographical conditions within which users reside, or do local cultural conditions continue to shape individuals' views toward feminism expressed on social media?

\section{METHODS}

In this section, we describe the methods used to operationalize local place character, calculate Twitter sentiment toward feminism, and analyze the relationship between these variables. Because topic modeling has not been widely used in the study of local culture and public opinion, we provide an extended discussion of our use of this method to measure locations' place character. Then, we explain the methods involved in the collection and classification of tweets about feminism before reviewing the analytic approach used to examine the relationship between local place character and online support for feminism.

\section{Measuring Place Character}

In defining place character, cultural sociologists have examined narratives of places describing their popular reputations, collective values, aesthetics, and characterizations of the type of people who reside there (Molotch et al. 2000; Paulsen 2004). These aspects of culture are not easily quantified. As a result, the majority of research on place character has used qualitative methods comparing a small number of select locations (Boren 2006; Brown-Saracino 2017; Molotch et al. 2000). These studies have examined place character through the narratives people use to describe their community as well as through the collection of murals, billboards, and written materials that convey attributes of a particular area (Molotch 2002). Focusing on local 
cultural features that emerge in this collection of place narratives, previous research has significantly advanced our understanding of how place character shapes social life. However, ethnographic approaches provide less information on more generalizable trends, which require data on variability in place character across a larger number of areas.

Drawing from advances in computational social science over the past decade, this study builds from previous qualitative work by generating quantified measures of place character through extracting themes from a corpus of documents providing rich descriptions of local reputation. A crucial task in this endeavor is the development of such a corpus of cultural reputations. In identifying areas where place character is explicitly conveyed, Paulsen (2004: 249) states, "accounts by travel writers and journalists can provide insights into how a place is represented to a broad audience." Drawing on this approach, we use descriptions of U.S. cities from the Lonely Planet online travel guide. Beyond providing information on accommodations and lodging, online guidebooks also provide narrative descriptions of places' cultural reputation. For example, the Lonely Planet page for Madison, WI focuses heavily on the area's reputation as a liberal college town, illustrated in the following excerpt:

Madison reaps a lot of kudos - most walkable city, best road-biking city, most vegetarian-friendly, gay-friendly, environmentally friendly and just plain all-round friendliest city in the USA. Ensconced on a narrow isthmus between Mendota and Monona Lakes, it's a pretty combination of small, grassy state capital and liberal, bookish college town.

Instead of focusing primarily on Madison's hotels and restaurants, the Lonely Planet entry centers more affective attributes which convey values and aesthetics. Surely, this broad description may not resonate with the lived reality of all Madison residents, but it likely 
corresponds to how these individuals may perceive their city's broader reputation. In other words, these descriptions convey place narratives - "the stories a city tells about who it is" (Brown-Saracino 2017: 198). As previous research has shown, narratives of place character have an impact on individuals' sense of identity even if they do not perceive their local environment as perfectly aligning with these broad characterizations (Correll et al. 2017). Like previous qualitative research, we operationalize place character through focusing on the narratives used to describe cultural reputations. Our use of travel guides, as opposed to interviews or ethnography, allows us to examine a larger number of locations and explore more general trends regarding the relationship between place character and support for feminism.

Online travel guide descriptions provide a useful corpus of place narratives ideal for the purposes of our study. There are, however, important limitations. Travel guides descriptions are oriented to tourists and, as a result, may focus less on local inequalities and the drawbacks of cities for certain groups of residents. Because most U.S. cities are characterized by high levels of racial inequality and residential racial segregation (Massey and Denton 1993), it is highly possible that there exists racial differences in residents' feelings about place and perceptions of local culture (see Hunter and Robinson 2018). As Whites tend to reside in more economically advantaged neighborhoods with greater access to cultural activities (Quillian 2012), it is further possible that travel guide descriptions are biased toward White residents' experiences while resonating less with those of Black, Latinx, and/or Asian residents. Along similar lines, readers of travel guides are likely those with the means and disposable income for recreational vacations. Consequently, the narratives provided may emphasize cultural features more salient to those in middle/upper classes, while being less relevant to working class individuals. We use Lonely Planet guides because they are the most widely read travel guide in the world (Iaquinto 2011) 
and therefore may provide more commonly known characterizations. A valuable contribution of future research may compare place narratives across multiple travel guides or additional sources of city descriptions to examine how local characterizations differ according to intended readership spanning different racial and socio-economic backgrounds.

Leveraging the documentation of place narratives in travel guides, we collected commentaries for cities across the U.S. from Lonely Planet's online guidebook. We selected principal cities corresponding to U.S. labor sheds constituting all commuting zones in the U.S. (Fowler et al. 2019). We focused on commuting zones, rather than city boundaries, because our interest is in describing cultural environments that affect all people who live and work in a particular area. 133 of the 179 total U.S. commuting zones contained cities that were featured in Lonely Planet. For each of these pages, we extracted all commentary text providing descriptive overviews of cities using the rvest package in r (Wickham 2019). We did not collect additional text pertaining to accommodation, restaurants, or activities, as we were primarily interested in the descriptive content that conveyed a cohesive narrative about each place's character.

We used latent Dirichlet allocation (LDA) (Blei et al. 2003; DiMaggio et al. 2013) to perform topic modeling on the corpus of cities' Lonely Planet pages in order to identify common themes across texts. LDA identifies themes (otherwise referred to as topics) that exist in a collection of texts through examining patterns of word co-occurrence within documents in relation to their distribution across a corpus of documents as a whole. Collections of words commonly found together are used to infer the presence of topics, which are heterogeneously distributed across documents. For example, a single document may consist of 20 percent one topic, 30 percent a second topic, and 50 percent a third topic. This approach provides a more 
realistic method for modeling aspects of place character, which are less likely to be discrete and more likely to share multiple themes in varying degrees (Molotch 2002).

Before applying LDA to the corpus of city descriptions, we removed stop words (commonly used words without substantive meaning, such as "the," "a," and "in") and names for states and cities. We also stemmed words to focus on common derivatives (e.g. "excitement" and "exciting" are stemmed to "excit"). We then conducted multiple independent LDA models with an increasing number of topics. We identified the ideal number of topics by comparing the average probabilistic coherence across topics calculated from the probability that words occur together within a topic relative to their presence in the full corpus. We focus on this model fit statistic because coherence best reflects the interpretability of topics (Hinneburg et al. 2014; Roder et al. 2015). We report average topic coherence for each LDA model in Figure 1. With the exception of the four-topic model, coherence improves from one through five topics, after which improvements in coherence are unstable and less pronounced. This suggests that the five-topic model provides the most parsimonious representation of topics included in the corpus of city descriptions. Therefore, we selected the LDA model with five topics to represent the ideal framework for describing local place character. We validated our selection of this model with three additional fit indices (perplexity, topic density, and word-to-topic probabilities), all of which suggested that the five topic solution ideally represented the different ways in which cities are described in Lonely Planet travel guide descriptions. 
Figure 1. Probabilistic Coherence Across Topic Specifications

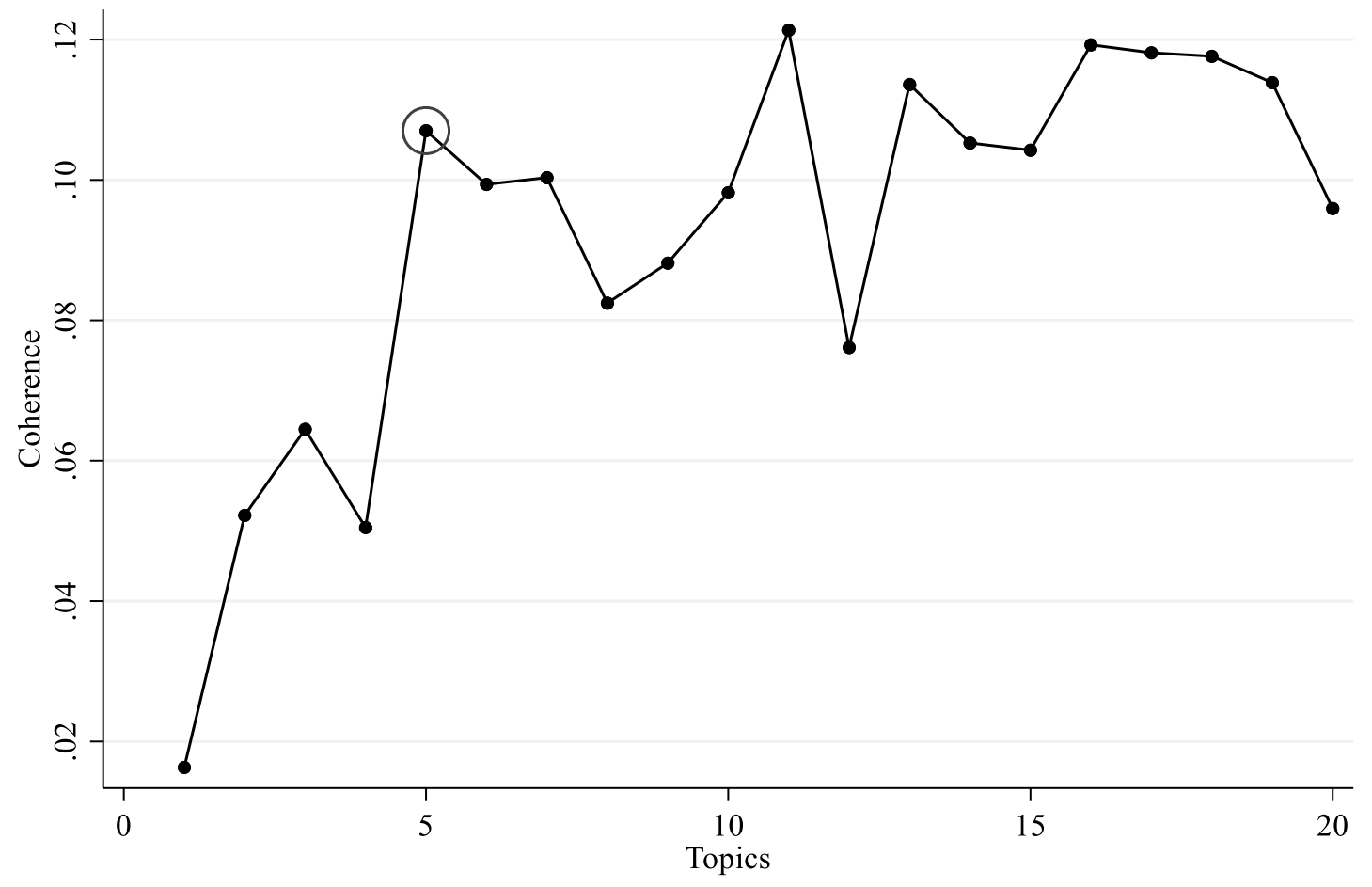

Note: The circled point represents the selected LDA model with five topics.

Topics are assigned meanings based on substantive interpretation of the most frequent words and the distribution of topics across cities. Figure 2 presents word clouds for the fifty most common word stems found in each topic. Word stems in Figure 2 are weighted so that larger and more central word stems have a higher probability of being found in the topic relative to other topics. Because topic identification is a function of word co-occurrence, interpretation of word clouds should consider the collection of items associated with a topic instead of stressing the importance of single word stems. In addition to the word clouds in Figure 2, Table 1 reports cities with the highest proportion of each topic. 
Figure 2. Word Clouds Describing Place Character Topics

Topic 1: Revitalization brick today ecom remain stretch drink bestartcall industri localold artis found creativeat $900 d_{\text {garden }}$ world trail indi $^{\text {citi }}$ past penninclud bUIld lotcapit earli MUSEUM

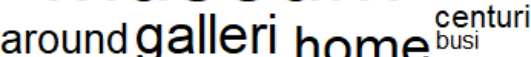

(busi

biggest nation cafe boom nearbinktsight place $_{\text {move }}$ ithout
Topic 2: Gastro Hub

breweri

footbal marketpolit

turn home hard craft

lower beer nation hike central first fair central first lifemaketopciti proxim trail fOOd made rest find 1000 feel hour get year stop art ${ }^{\text {thank }}$ plus small excel fresh Small around work capitol decadthree ${ }^{\text {rang }}$ museum public
Topic 3: Local Hub outdoorsurround mountain visitor near state neighborhood plenti line natur attract park pretti univers rivershop tourist stillarea rivershop offer

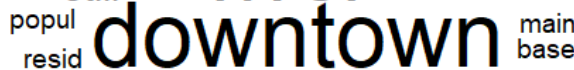
${ }_{\text {dine }}$ bar center live citi space two mile restaur capit name sprawl estaur district easi Scene along far explor largest major now recent vịbrant hotel

\section{Topic 4: Vibrant}

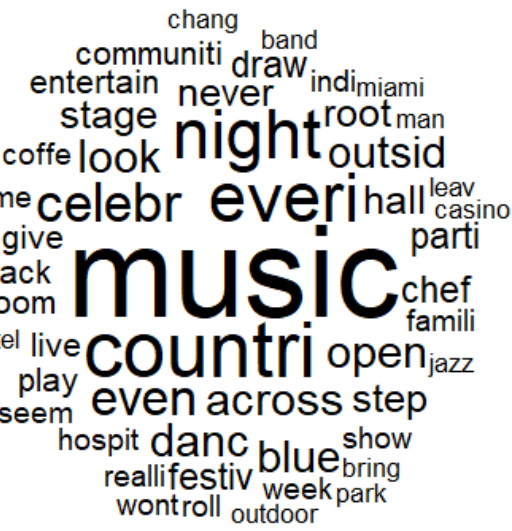

Topic 5: History \& Arts

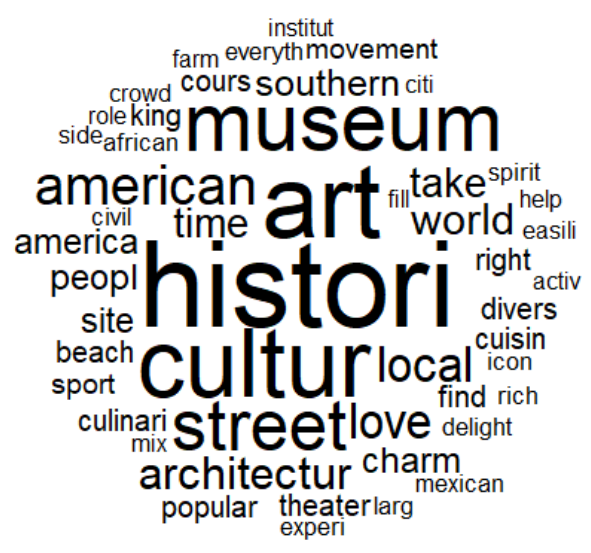


Table 1. Top 10 Cities by Dimensions of Place Character

\begin{tabular}{|c|c|c|c|c|c|}
\hline \multirow[b]{3}{*}{ Rank } & \multicolumn{5}{|c|}{ Topics } \\
\hline & 1 & 2 & 3 & 4 & 5 \\
\hline & Revitalization & Gastro Hub & Local Hub & Vibrant & History \& Arts \\
\hline 1 & Dayton, $\mathrm{OH}$ & South Bend, IN & Tallahassee, FL & Nashville, TN & Kansas City, MO \\
\hline 2 & Albany, NY & State College, PA & Sioux Falls, SD & Las Vegas, NV & Montgomery, AL \\
\hline 3 & Helena, MT & Billings, MT & Bismarck, ND & Austin, TX & Boston, MA \\
\hline 4 & Wichita, KS & Lubbock, TX & Huntsville, AL & Lafayette, LA & Corpus Christi, TX \\
\hline 5 & Raleigh, NC & Fresno, CA & Traverse City, MI & Cleveland, $\mathrm{OH}$ & Rochester, NY \\
\hline 6 & Pendleton, OR & Omaha, NE & Rapid City, SD & Wenatchee, WA & Atlanta, GA \\
\hline 7 & Minneapolis, MN & Milwaukee, WI & Dover, DE & Miami, FL & Philadelphia, PA \\
\hline 8 & Paducah, KY & Portland, OR & Little Rock, AR & New Orleans, LA & Dallas, TX \\
\hline 9 & Columbia, MO & Lincoln, NE & Roanoke, VA & Bend, OR & Orlando, FL \\
\hline 10 & Abilene, TX & Columbus, $\mathrm{OH}$ & Clarksburg, WV & Charleston, SC & Bangor, ME \\
\hline
\end{tabular}


The first topic includes a number of frequent word stems related to arts, creativity, and aesthetics, such as "galleri," "creativ," "garden," "artist," "museum," and "art." In addition, there are also several words related to manufacturing or industrial origins, including "build," "industri," "brick," and "past." The first topic is also the most common in locations such as Dayton, $\mathrm{OH}$ and Paducah, KY that have historically had economic declines associated with deindustrialization, but have experienced growth in recent decades driven by booming art and cultural centers. Collectively, we characterize these attributes as portraying a place character of "Revitalization" to describe cities' reputations as areas that had previously experienced economic decline, but are now undergoing resurgence based largely in the creative arts. ${ }^{4}$

The second topic to emerge from LDA included a combination of words related to culinary scenes as well as active lifestyles. For example, some of the most salient word stems are "food," "breweri," "beer", "trail," "hike," and "bike." This topic is most common in mid-size locations known for thriving breweries such as Milwaukee, WI and South Bend, IN. We label this topic as referring to a place character of "Gastro Hubs." The third topic also contains a number of frequent word stems related to consumption, but differs in that these items characterize more conventional forms of city centers including words such as "downtown" and "shop." In contrast to the place character of Gastro Hub (the second topic) that described local culinary scenes using words such as "breweri," "craft," and "beer," the third topic features more

\footnotetext{
${ }^{4}$ We acknowledge that revitalization is a "broad and malleable term, imbued with politically charged meanings," which is most often used colloquially to refer to place-based retail and infrastructure investments, rather than other forms of urban-revitalization policies, such as equity-based programs designed to support individuals and families (Grodach and Ehrenfeucht 2016:6). Thus, we adopt this term, in part, to signal its colloquial meaning, which is reflected in the travel guide narratives that portray particular areas as "revitalizing."
} 
traditional words such as "bar" and "restaurant," suggesting that these locations have a more customary place character. We therefore refer to this aspect of place character as "Local Hubs."

A fourth topic included word stems conveying a place character of entertainment, such as "music," "night," “celebr," "danc," "live," "festiv," and "parti." This topic is most prevalent in locations like Nashville, TN, Las Vegas, NV, and Miami, FL that are widely regarded for music, dancing, and nightlife. One exception is Wenatchee, WA, which was found to have a high prevalence of this topic, but is not largely known for music and entertainment. Although Wenatchee is not an entertainment hub, it is the largest apple producer in the country. Lonely Planet used language such as "the varieties are endless" and "eye-catching" to describe the apple orchards in this location in a similar manner as it described the nightlight in Miami and Nashville. This dynamic signifies a limitation of LDA, which is unable to identify the context of word usage, but instead relies on patterns of co-occurrence. Despite this limitation, there remains a general trend for word frequencies and cities associated with the fourth topic to emulate a "Vibrant" place character.

The fifth topic to emerge in LDA included word stems associated with history and culture. These included "histori," "cultur," “art," "museum," "architectur," "theater," and "icon." Additionally, this topic is most prevalent in cities that are well-known for local history (Montgomery, AL, Boston, MA) and others with reputed museums and cultural centers (Atlanta, GA and Dallas, TX). Although this topic shares an emphasis on the arts with the topic of "Revitalization," it differs from the first topic in that it does not emphasize art as connected to economic resurgence, but more of a durable feature of the history of the place. We refer to this topic as reflecting a place character of "History and Arts." 
In total, our application of LDA to Lonely Planet city descriptions revealed five aspects of place character: Revitalization, Gastro Hubs, Local Hubs, Vibrancy, and History/Arts. The strength of LDA is that it reduces the complexity of cultural reputations into five discernable categories that can be used to draw generalizable patterns across the U.S. The limitation, however, is that it simplifies the richness and complexity of local place character. Yet, there already exists a large body of qualitative studies providing in-depth descriptions of local culture (Brown-Saracino 2017; Guenther 2010; Molotch et al. 2000). Less attention has been given to broad trends and their relation to other social phenomena. Thus, in this study the tradeoff of depth for breadth fills a gap in our existing knowledge on the relationship between local cultural conditions and online public discourse towards social issues such as feminism.

Our application of LDA conceptualizes each city's place character as being constituted by the composition of the five topics described above. This approach allows us to quantify place character as a set of continuous variables. The place character of Houston, TX, for example, is $60 \%$ Local Hub, 21\% Vibrant, 12\% History \& Arts, 7\% Gastro Hub, and 0\% Revitalization. The most prominent aspect of place character in Houston is its reputation as a Local Hub. Yet, this is not all that Houston is known for. By conceptualizing cities' place character as constituted by up to five components with varying levels of salience, our approach captures some complexity to the extent that place character is composed of multiple dimensions. Thus, our use of LDA produced five separate variables measuring the salience of different aspects of place character in cities across the U.S. We use these components in the analysis below as predictors of online Twitter sentiment toward feminism. 


\section{Online Twitter Sentiment toward Feminism}

To examine online support for feminism, we collected a sample of tweets containing words related to feminism and classified them as supportive or in opposition to feminist ideals. We used the search Twitter API to collect tweets. We intentionally collected tweets taking place during Mother's and Father's Day weekends in 2017 because these cultural events lent themselves to increased discussion about feminism as advocates used the occasion to celebrate feminist parents or feminist ideals, while opponents of feminism critiqued the movement for harming the family form. In identifying terms to use in the search Twitter API, we followed standard practices (Liu 2015) and used WordNet's lexical database of word relations (Princeton University 2010). We first identified words related to the term "feminism" and used the results in additional searches until reaching saturation. In total, this produced eight words (feminist, feminism, women's rights, women's rightist, women's liberationist, women's libber, women's liberation, and women's lib). Because Twitter users often omit punctuation, we also included versions of words that did not contain apostrophes. ${ }^{5}$ We limited our key terms to those related to feminism because it is widely recognizable and debated. However, a limitation is that our search terms did not include references to intersectionality or queer politics and, therefore, our findings may underrepresent users who more commonly discuss feminism within those frameworks. Applying the search terms for feminism in the search Twitter API, we collected 118,793 tweets from users with locations stated in their profile that we use to link to cities' commuting zones. ${ }^{6}$ This initial sample included only users with credible locations referring to actual places, omitting

\footnotetext{
${ }^{5}$ We did not include misspellings of words because this produced too many possibilities and using correct spelling offered a more standardized approach.

${ }^{6}$ Our omission of tweets from users without geo-coded profiles limits the representativeness of our study. Yet, because this sampling bias is consistent across geography, the data remain appropriate for geospatial comparison (Widener and Li 2014).
} 
those listing fictional locations (e.g. "Wanderlust" or "Somewhere Over the Rainbow") as well as those with locations that were unable to be identified with google maps. We used botometer to identify and remove 4,099 bot accounts associated with 13,727 tweets. $^{7}$ The proportion of bots in our sample of tweets is similar to what has been found in previous research (Varol et al. 2017). After removing bots, the sample included 105,066 tweets.

We used both qualitative and naïve Bayes classification to classify tweets as supporting or opposing feminism (Nelson 2017). The political nature of feminism lent itself to dichotomous coding, as users clearly expressed opposition or support for feminist ideals or feminism in their posts. Of the 105,066 tweets, about half $(51,562)$ were retweets consisting of 3,605 unique texts. We qualitatively coded these tweets as either positive or negative toward feminism. The remaining 53,504 tweets were unique texts. For these, we used naïve Bayes classification (Jurafsky and Martin, forthcoming; Scarborough 2018) which classifies tweets based on the probability that associated words are found in a positive or negative tweet. To train the naïve Bayes classifier, we used a randomly selected five percent sample of tweets from the group of 53,504 unique texts to serve as a training set. We qualitatively coded tweets in the training set as either positive or negative. Then, we removed stop words and tokenized tweets into one and twoword tokens used to generate positive and negative lexicons. ${ }^{8}$ Each lexicon contained tokens that were assigned probabilities of being in a negative or positive tweet based on their prevalence in each group within the training set. We used these lexicons in applying the naïve Bayes classifier

\footnotetext{
${ }^{7}$ Botometer (https://botometer.iuni.iu.edu/\#!/) calculates the probability that Twitter accounts are bots. Accounts with a probability of .5 or greater were identified as bots (Varol et al., 2017). Accounts not identified with botometer were labeled as bots if they were no longer on Twitter six months after the initial web scraping because Twitter removes accounts suspected of being bots. ${ }^{8}$ Single-word and negations were used as tokens in pre-testing, but did not perform as well in accuracy as the one and two-word tokenizing.
} 
to the full sample of unique tweets. This approach classified tweets as positive if the product of positive token probabilities and the prior (determined as the proportion of positive and negative tweets in the training set) was greater than the product of negative token probabilities and the prior. To assess performance, we compared the results of the naive Bayes classifier to a randomly selected sample of 100 tweets that were qualitatively coded. 74.49 percent of these tweets were correctly coded, which is on par with sentiment classifiers used in previous research (Go et al. 2009; Pang et al. 2002). Combining the qualitatively and naïve Bayes classified tweets resulted in a fully coded dataset of 105,066 tweets. In total, $73.7 \%$ of tweets were coded as supporting feminism, $25.7 \%$ were coded as opposing feminism, and $.6 \%$ were coded as missing because they contained only username tags. Table 2 provides an illustrative example of positive and negative tweets included in the sample.

We merged tweets to cities based on commuting zones defined by the Bureau of Economic Analysis's labor sheds (Fowler et al. 2019). We chose this level of aggregation because it accounted for the fact that cultural reputations do not necessarily end at city boundaries, but instead relate to the broader population of people who live, work, and frequent the areas within and around a principal city. Twitter users were matched to commuting zones based on their stated location in their profile. We use profile locations, rather than geotagged tweets, because research indicates profile locations better reflect users' place of residence (Alex et al. 2016). When calculating the percentage of tweets that are positive toward feminism, we followed a previous approach (Scarborough 2018) and weighted tweets to adjust for the fact that single users accounted for multiple tweets $\left(U \operatorname{ser} W T_{i}=\frac{1}{\text { tweets }_{i}}\right)$. The resulting estimate represents the user-adjusted percentage of tweets supporting feminism. 133 commuting zones (of 
179) were represented in the Lonely Planet data on cultural reputations. Of these, one had less than 10 tweets (Minot, ND) and was dropped.

Table 2. Illustrative Sample of Tweets

\begin{tabular}{|c|c|}
\hline \multicolumn{2}{|c|}{ Coded as Positive } \\
\hline 1 & my mom and i are watching @HandmaidsOnHulu together and discussing feminist theory \\
\hline 2 & Thank you to my fellow kick-ass feminist mom! \\
\hline 3 & $\begin{array}{l}4 \text { Year Old: I'm a GIRL doctor! Husb: Or as WE say, a doctor \#dudefeminist \#feminist } \\
\text { \#raisinggirls \#momlife }\end{array}$ \\
\hline 4 & $\begin{array}{l}\text { Honest feminist support of moms would be an all inclusive solidarity, regardless if you } \\
\text { agree w parenting choices (or situations). }\end{array}$ \\
\hline 5 & $\begin{array}{l}\text { Joni Mitchell, Emmylou Harris, Eudora Welty...moms. Hear more about feminist icons } \\
\text { here }\end{array}$ \\
\hline 6 & $\begin{array}{l}\text { I LOVE my dad but having my moms last name was always kinda badass and cemented } \\
\text { my feminism. }\end{array}$ \\
\hline 7 & My dad is such a feminist and he doesn't even know it \\
\hline 8 & $\begin{array}{l}\text { today i made my dad realize how much of a feminist he is and it's the best thing that } \\
\text { happened to me today }\end{array}$ \\
\hline 9 & Y'all, my dad is awesome \#feminist \#nastywoman \#heforshe \#lovemydad \\
\hline 10 & I am a proud feminist father \\
\hline
\end{tabular}

Coded as Negative

11 As a yogi and a mom, when i became a mom, feminism let me down.

12 3-yr-old boy wants to play with boy stuff, but his feminist mom makes BIG point she won't tolerate THAT...

13 Smh girls with puppies out here calling themselves mom's, surprised there hasn't been a feminist rant yet

14 Women's Rights must feed off of the blood of there non existent children to work, a cost way to high for any civilized culture

15 Idc what these feminist say.no kid boy wants to hear about their mom ass naked on the internet from their peers

16 Imagine having a mom with a nose ring. Feminism was a mistake.

17 feminism worst thing ever happened to families, to children

18 why does it seem like every feminist had a terrible father

19 Feminists are tired of the emasculated manchildren that feminism created

20 Feminism is a war on men. Motherhood and child bearing

Note: Tweets edited to remove usernames and identifying information. 


\section{Analytic Models}

We use OLS regression models to test the relationship between local place character and online support for feminism. As noted above, one advantage of using topic modeling is that it treats topics (components of place character in the present study) as making up different proportions of individual texts (or the total place character of cities). Thus, the LDA used above generated five continuous variables representing shares of cities' place character. The sum of all five variables equals one for all areas, with varying proportions for each. To account for the nonindependence of variables measuring place character, we use five equations modeling cultural reputations separately as key predictors. This approach shows the effect of one aspect of place character in relation to areas' average of the four additional components. ${ }^{9}$ Each model also includes a number of control variables. To account for the relationship of education and presence of universities with support for feminism, we control for the proportion of residents with a college degree. Since younger individuals tend to be more supportive of feminism and gender equality (Scarborough et al. 2019), we also control for the percentage of the local population aged 20 to 30 years old. Racial composition is controlled for through a variable measuring the percentage of the population that is White. Finally, we account for urbanicity by including a variable measuring the (logged) population. All control variables were generated using data from the 2017 American Community Survey five-year sample.

\section{RESULTS}

Descriptives

\footnotetext{
${ }^{9}$ It is possible that the combination of different aspects of place character is also associated with online sentiment toward feminism. However, our use of LDA treats components of place character as non-independent. Therefore, we apply them in separate models and do not explore interactions.
} 
Table 3 reports descriptive statistics for all areas included in the sample. On average, over three-quarters of tweets were positive toward feminism. In the least supportive areas of the U.S. (e.g. Corpus Christi, TX), about $60 \%$ of tweets related to feminism were positive, while in the most supportive areas (e.g. Burlington, VT) the vast majority were positive. The fact that over $50 \%$ of tweets were positive toward feminism in all places included in this study indicates that public discourse on Twitter is mostly supportive of feminism. Nonetheless, there exists meaningful variation in levels of support.

Table 3. Descriptive Statistics

\begin{tabular}{lrrrr}
\hline & Mean & SD & Min & Max \\
\hline $\begin{array}{l}\text { \% Positive Tweets Toward Feminism } \\
\text { Dimensions of Place Character }\end{array}$ & $77.6 \%$ & 6.4 & 59.6 & 96.9 \\
$\quad$ Revitalization & $20.8 \%$ & 20.0 & 0.0 & 84.3 \\
Gastro Hub & $18.9 \%$ & 17.9 & 0.0 & 65.8 \\
Local Hub & $32.8 \%$ & 20.7 & 0.1 & 83.1 \\
Vibrant & $11.3 \%$ & 15.9 & 0.0 & 62.5 \\
History \& Arts & $16.1 \%$ & 17.2 & 0.1 & 74.9 \\
Socio-Demographic Characteristics & & & & \\
\% College Educated & $27.8 \%$ & 5.7 & 16.8 & 43.7 \\
\% of Population 20-30 Years Old & $14.0 \%$ & 1.6 & 9.8 & 19.5 \\
\% White & $67.6 \%$ & 17.9 & 7.2 & 94.0 \\
Population & $2,230,712$ & $3,351,198$ & 145,885 & $23,815,321$ \\
\hline
\end{tabular}

iThe median population in the sample is $1,129,972$.

Aspects of place character were well distributed across the sample. The most widely shared was "Local Hub." Across cities, an average of about 33\% of areas' place character had to do with its status as a local hub. In contrast, only $11 \%$ of areas' average place character were related to Vibrancy, suggesting that this cultural reputation is more restricted to certain locations. The remaining three components each made up an average of approximately one-fifth of local place character, with about $21 \%$ related to Revitalization, $19 \%$ pertaining to Gastro Hub, and 
$16 \%$ reflecting cultural reputations for History and Arts. Examining the range of these place character attributes, there were areas where each aspect was absent as well as locations where it made up at least $60 \%$ of areas' overall character. This suggests that many places possess a particularly salient cultural reputation reflected by one of the five topics that emerged in LDA.

Table 3 also reports descriptives for socio-demographic control variables. On average, just over a quarter of commuting zone residents had a college degree, and $14 \%$ were young adults aged 20 to 30 years old. The average racial composition of commuting zones in the sample was about two-thirds white, and the average population was over 2.2 million, with the median population at 1.1 million.

\section{Cultural Reputations and Support for Feminism Online}

Table 4 reports the results of regression models testing the relationship between place character and the percentage of tweets that are positive toward feminism. Examining control variables, the percent of residents with a college degree was a powerful predictor of online support for feminism. Across all models, more educated areas were found to have a higher percentage of positive tweets toward feminism $(\mathrm{p}<.001)$. No other control variables were found to be significantly related to online support for feminism. 
Table 4. OLS Models Predicting Percentage of Positive Tweets Toward Feminism

\begin{tabular}{|c|c|c|c|c|c|}
\hline & Revitalization & Gastro Hub & Local Hub & Vibrant & $\begin{array}{l}\text { History \& } \\
\text { Arts }\end{array}$ \\
\hline \multirow[t]{2}{*}{$\%$ College Educated } & $0.376 * * *$ & $0.394 * * *$ & $0.363 * *$ & $0.389 * * *$ & $0.409 * * *$ \\
\hline & $(0.110)$ & $(0.113)$ & $(0.112)$ & $(0.113)$ & $(0.113)$ \\
\hline \multirow[t]{2}{*}{$\%$ Aged $20-30$} & 0.116 & -0.018 & 0.078 & 0.014 & -0.023 \\
\hline & $(0.356)$ & $(0.366)$ & $(0.360)$ & $(0.365)$ & $(0.364)$ \\
\hline \multirow[t]{2}{*}{ Population (logged) } & -0.571 & -0.794 & -1.017 & -0.685 & -0.638 \\
\hline & $(0.594)$ & $(0.609)$ & $(0.614)$ & $(0.616)$ & $(0.609)$ \\
\hline \multirow[t]{2}{*}{$\%$ White } & 0.026 & 0.021 & 0.032 & 0.025 & 0.018 \\
\hline & $(0.033)$ & $(0.035)$ & $(0.034)$ & $(0.034)$ & $(0.035)$ \\
\hline \multicolumn{6}{|c|}{ Dimensions of Place Character } \\
\hline \multirow[t]{2}{*}{ Revitalization } & $0.072 * *$ & & & & \\
\hline & $(0.026)$ & & & & \\
\hline \multirow[t]{2}{*}{ Gastro Hub } & & 0.027 & & & \\
\hline & & $(0.030)$ & & & \\
\hline \multirow[t]{2}{*}{ Local Hub } & & & $-0.056^{*}$ & & \\
\hline & & & $(0.027)$ & & \\
\hline \multirow[t]{2}{*}{ Vibrant } & & & & -0.019 & \\
\hline & & & & $(0.035)$ & \\
\hline \multirow[t]{2}{*}{ History \& Arts } & & & & & -0.044 \\
\hline & & & & & $(0.033)$ \\
\hline \multirow[t]{2}{*}{ Constant } & $70.21 * * *$ & $76.03 * * *$ & $80.27 * * *$ & $74.68 * * *$ & $74.93 * * *$ \\
\hline & (10.79) & (10.97) & (11.07) & (10.96) & $(10.88)$ \\
\hline $\mathrm{N}$ & 132 & 132 & 132 & 132 & 132 \\
\hline R-sq & 0.162 & 0.118 & 0.141 & 0.114 & 0.124 \\
\hline
\end{tabular}

Standard errors in parentheses

* $\mathrm{p}<0.05 ; * * \mathrm{p}<0.01 ; * * * \mathrm{p}<0.001$

Examining the relationship of place character to online support for feminism, the first model shows that areas known for having a reputation for Revitalization were more supportive of feminism $(\mathrm{p}<.01)$. This relationship is illustrated in Figure 3a. 76.8\% of tweets about feminism are predicted to be positive in places where the local place character was only $10 \%$ related to Revitalization, while $81.8 \%$ are predicted to be positive in areas where the place character was $80 \%$ related to Revitalization. These findings indicate that feminism receives greater support 
online in areas known to be undergoing cultural change in the context of economic resurgence. Indeed, qualitative studies have often uncovered thriving feminist communities in the locations we identify as having a highly salient place character of Revitalization. Through interviews and ethnographic research, Ezekiel (2002) argues that feminism in Dayton, OH (84\% of place character related to Revitalization) not only receives greater public support, but is also more radical than forms of feminism existing in the better-known metros of New York and Los Angeles. Other research in Wichita, KS (67\% of place character related to Revitalization), has highlighted common support for feminist ideals among the city's active and politically influential LGBTQ community (Janovy 2018).

Figure 3. Relationship of Local Place Character to Online Twitter Sentiment Toward Feminism

3a. Revitalization to Online Sentiment

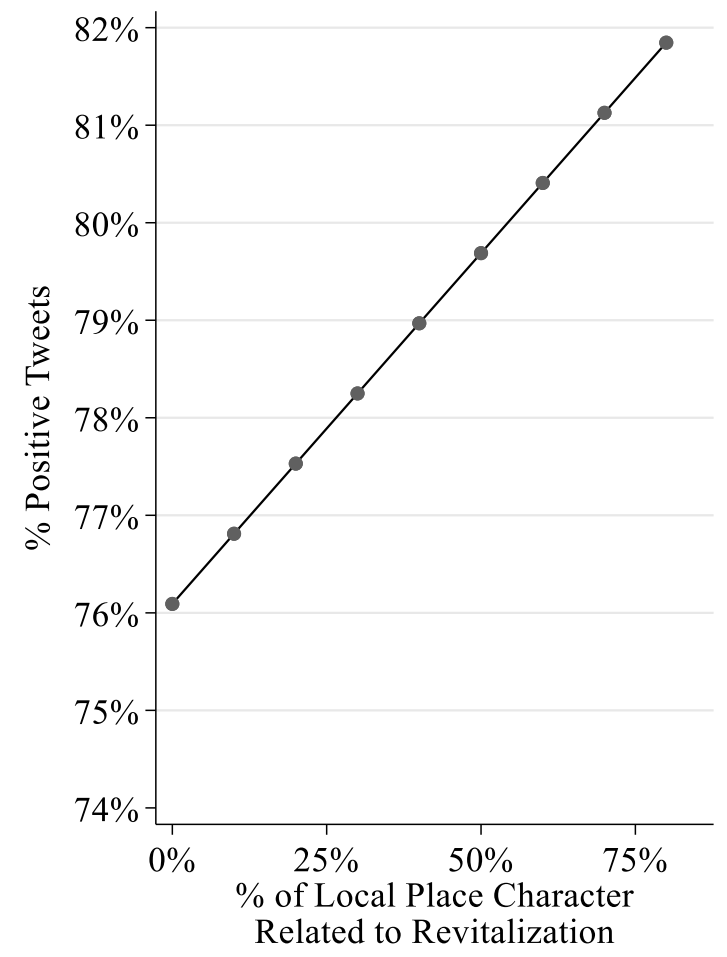

3b. Local Hub to Online Sentiment

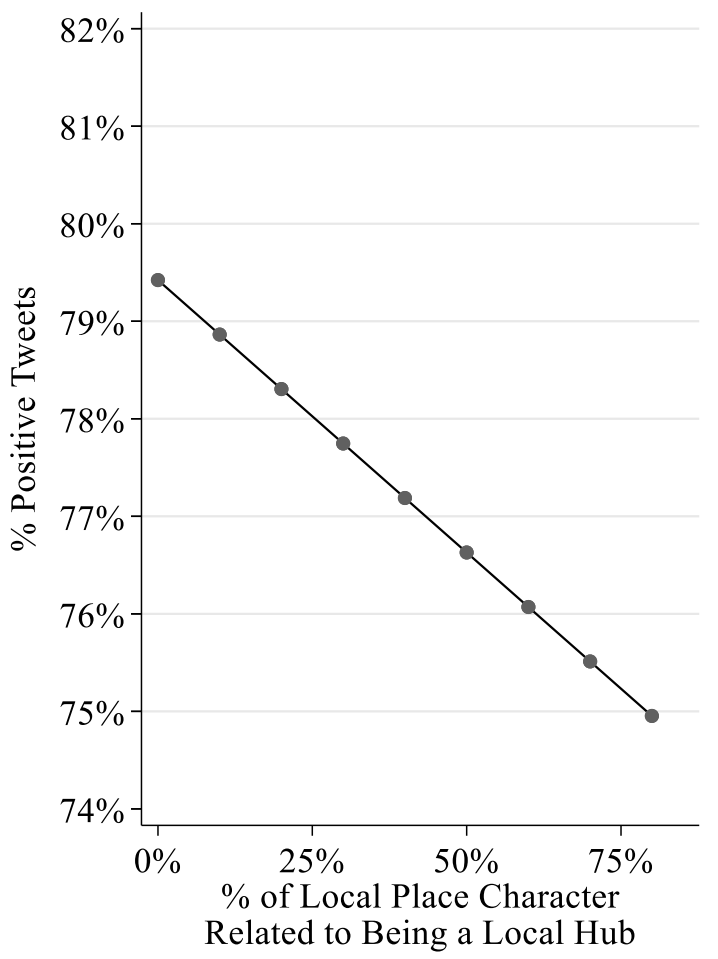


The second model tests the effects of having a place character as a Gastro Hub. The model reveals no association of this cultural aspect to online feminist discourse. Turning to the third model, however, the place character of a Local Hub was negatively associated with online support for feminism $(\mathrm{p}<.05)$. This relationship is illustrated in Figure $3 \mathrm{~b}$. Areas where the local cultural reputation is $10 \%$ related to being a Local Hub are predicted to have $78.9 \%$ of tweets with positive sentiment toward feminism, while $75 \%$ of tweets are predicted to be positive in areas where the local place character is measured as being $80 \%$ characterized as a Local Hub. This indicates that Local Hubs known as regional shopping centers are less supportive of feminism online. These findings provide generalizability to qualitative studies taking place in cities that we identify as Local Hubs. Research based in Tallahassee, FL (83\% of place character related to Local Hub status), for example, has found more widespread and vocal support for conservative movements such as the Tea Party (Rohlinger and Brown 2009). Tallahassee residents with more feminist or liberal views report feeling pressure to hide their opinions from neighbors and colleagues (Rohlinger et al. 2014). Our findings suggest similar dynamics are also occurring on Twitter in this location and others known as Local Hubs.

Next, Table 4 reports that Vibrancy was unrelated to Twitter sentiment, suggesting that nightlife and entertainment do not translate into greater support for feminism online. The last model in Table 4 explores the relationship between a local reputation of History/Arts to Twitter sentiment, revealing that this aspect of place character was unrelated to feminist discourse.

Taken as a whole, the results from Table 4 indicate that areas with cultural reputations as Gastro Hubs, Vibrancy, and History/Arts have about average Twitter sentiment toward feminism, net of control variables. Meanwhile, areas with a place character of Revitalization 
have more positive feminist discourse online, and areas known as Local Hubs have relatively more negative online Twitter sentiment.

\section{DISCUSSION}

Online discussions of feminism allow individuals with shared social interests to connect with one another regardless of physical distance. As a result, some have argued that opinions toward feminism are no longer tied to a particular geographical location, but instead transcend space to rally support across a wide range of contexts (Crossley 2015). While social media certainly provides the means to connect individuals across great distance, the results presented here indicate that local offline context continues to play a role in how feminism is discussed in online settings. More specifically, we found that Twitter posts were more positive toward feminism in areas of the U.S. portrayed by a place character of Revitalization - areas that once experienced economic decline but have had recent resurgence centered in creative activities and the arts. Twitter content was relatively more negative in areas of the country known as regional shopping hubs where individuals frequent restaurants and retail.

The connection of online Twitter sentiment to local place character resonates with research on offline feminist organizing that has found local place character to be highly influential in shaping levels of support for feminist ideals and activities occurring in public settings (Guenther 2010; Reger 2012). In other words, social movement discourse occurring on new forms of online media are shaped by similar geographical-cultural conditions as forms of offline discourse. Previous research has shown that online activism faces many similar challenges as activism occurring offline, such as measuring impact and strategizing effective demonstrations (Earl 2014). While the virtual domain of online settings would seem more resistant to the geographical constraints of local culture, our findings reveal that feminist 
discourse occurring on Twitter, as a visible and public form of social media, is nonetheless connected to the cultural environments where it originates in a similar way that offline discourse is rooted to local culture (Guenther 2010).

Our results also advance research on place character by suggesting that the influence of local culture extends beyond in-person dynamics to also shape views expressed online. Areas with a place character of Revitalization, such as Dayton, $\mathrm{OH}$ and Paducah, $\mathrm{KY}$, are presently undergoing broader change as artists and entrepreneurs relocate to these areas for cheap rent and urban appeal. While certainly smaller than Chicago or Los Angeles, these cities still contain urban scenes of old industrial buildings from their manufacturing past. The juxtaposition of old industry with new creative centers provides an eclectic vibe representing broader cultural change. In areas where the place character of Revitalization corresponds to gentrification and the displacement of long-term residents, disagreements between new residents (often artists and creative entrepreneurs [Lees et al. 2008]) and local advocates opposed to gentrification may further convey cultural values rooted in change, contention, and political mobilization (Grodach and Ehrenfeucht 2016). In such an environment of transformation, residents express greater online support for feminist ideals advocating for social change. In addition, the potential for a place character of Revitalization to coincide with gentrification also has implications on racial inequality when gentrification occurs in historically Black, Latinx, or Asian communities. In these settings, efforts to address racial inequities caused by gentrification may facilitate engagement with activism across social movements such as feminism. It is also possible, however, that new residents in gentrifying areas hold pro-feminist views online that do not translate to racial or economic justice activities in their community. Future research may build from our findings to explore these dynamics within cultural contexts of Revitalization. 
Whereas a place character of Revitalization fostered greater support for feminism on Twitter, individuals in areas known as Local Hubs expressed more negative sentiment toward feminism online. These places, like Little Rock, AR, and Tallahassee, FL are characterized by a slow pace of life and urban sprawl. Far from the milieu of urban revitalization, these places exude tradition and stability. In such settings, community values may be more resistant to change and, therefore, in conflict with the transformative ideals conveyed by feminism. Identifying with these values, residents of Local Hubs express greater opposition to feminism on Twitter. These dynamics mirror the mechanisms by which place character shapes offline sentiment toward feminism, where residents' personally held values and opinions are deeply influenced by their identification with the reputation of their community (Guenther 2010; Molotch et al. 2000).

In contrast, areas of the U.S. where other aspects of place character were more salient, such as Gastro Hubs, Vibrant entertainment centers, and History/Arts, have average levels of online feminist Twitter sentiment. These areas are neither highly supportive of feminism nor greatly opposing it. Instead, they are caught between geographically bound disagreements on social media occurring among relatively anti-feminist areas (Local Hubs) and the pro-feminist places enmeshed a place character of Revitalization.

Our findings highlight a relationship between local place character and online feminist discourse. There are, however, important limitations to note. First, our use of online travel guide descriptions to measure place character allowed us to examine this attribute across a large number of locations by reducing the complexity of local cultural conditions to what could be quantified through topic modeling. Future qualitative research on the places included in our analysis may provide greater depth on local culture and the mechanisms by which it shapes individuals' views expressed online. Additionally, our focus on discourse examines only one 
aspect of social movement engagement. Yet, previous research suggests that positive views toward feminism do not always translate to activism (Aaronson 2003). Future research may further explore the conditions through which online sentiment leads to deeper engagement with activism, and whether local contexts influence this level of participation.

\section{CONCLUSION}

In proposing the concept of place character, Paulsen (2004: 243) urged scholars to better understand "how locality matters" by more fully examining "how material and symbolic aspects of place work together to direct activity on the ground." Building on this tradition, our findings suggest that the material and symbolic aspects of place are also working to direct activity off the ground, in other words, in cyberspace. This finding carries important implications for social movement scholars by highlighting how online feminist discourse is shaped by the local geographic contexts within which users reside and generate content. At the same time, it is possible to theorize this relationship in the reverse: Activities and sentiments that take place online could also influence our social environments and understandings of community in offline, geographically-specific contexts. Recent research has found early evidence for this relationship (Helmuth 2019), but further studies are needed to uncover the dynamic interaction of offline and online contexts in local communities. While it goes beyond the scope of the present study, future research could investigate how users' exposure to feminist sentiments online might influence the ongoing construction of local place character. We join others who have suggested that we cannot fully understand our local contexts_-or in the present case, local place character-without including cyberspace as part of the field site (Hallet and Barber 2014).

In many ways, the internet is redefining the way social movements gather support and influence society. Yet, a key finding from this study is that social movement discourse taking 
place on the internet is still very much connected to the geographical context from which online content is produced. Far from transcending space, discussions on social media websites like Twitter may make the cultural boundaries between places more apparent, as opinions from those residing in different areas more often come into contact and conflict. Although online discussions and social media provide the technological capability to transcend geographical distance, our findings illustrate how the cultural features of offline environments where individuals reside shape expressions made in online settings. As we continue to navigate the growing influence of online social movement discourse, it is important to remain grounded in the physical world where individuals cannot simply log off or shut down their profile, but instead must engage with and interpret cultural environments on a daily basis.

\section{REFERENCES}

Abelson, Miriam J. 2019. Men in Place: Trans Masculinity, Race, and Sexuality in America. Minneapolis, MN: University of Minnesota Press.

Alex, Beatrice, Clare Llewellyn, Claire Grover, Jon Oberlander, and Richard Tobin. 2016. "Homing in on Twitter Users: Evaluating an Enhanced Geoparser for User Profile Locations." Proceedings of the Tenth International Conference on Language Resources and Evaluation L16-1622: 3936-3944.

Blei, David M. Andrew Y. Ng, and Michael I. Jordan. 2003. "Latent Dirichlet Allocation.” The Journal of Machine Learning Research 3: 993-1022.

Brown-Saracino, Japonica. 2017. How Places Make Us: Novel LBQ Identities in Four Small Cities. Chicago, IL: University of Chicago Press.

Borer, Michael Ian. 2006. "The Location of Culture: The Urban Culturalist Perspective.” City and Community 5(2): 173-197.

Correll, Shelley, Cecilia L. Ridgeway, Ezra W. Zukerman, Sharon Jank, Sara Jordan-Bloch, and Sandra Nakagawa. 2017. "It's the Conventional Thought that Counts: How Third-Order Inference Produces Status Advantage." American Sociological Review 82(2): 297-327.

Crossley, Alison Dahl. 2015. "Facebook Feminism: Social Media, Blogs, and New Technologies of Contemporary US Feminism." Mobilization 20(2): 253-268.

Crossley, Alison Dahl. 2017. Finding feminism: Millennial Activists and the Unfinished Gender Revolution. New York: New York University Press. 
Place Character and Online Support for Feminism

Crossley, Alison Dahl. 2019. "Online Feminism is Just Feminism: Offline and Online Movement Persistence." In Jo Reger (ed.), Nevertheless, They Persisted: Feminisms and Continued Resistance in the U.S. Women's Movement: pp 60-78. New York: Routledge.

Crossley, Alison Dahl, and Laura K. Nelson. 2018. "Feminists Reshaping Gender." In Barbara J. Risman, Carissa Froyum, and William J. Scarborough (eds.), Handbook of the Sociology of Gender: pp. 549-559. New York: Springer.

DiMaggio, Paul, Manish Nag, and David Blei. 2013. "Exploiting Affinities between Topic Modeling and the Sociological Perspective on Culture: Application to Newspaper Coverage of U.S. Government Arts Funding." Poetics 41(6): 570-606.

Earl, Jennifer. 2014. "Something Old and Something new: A Comment on "New Media, New Civics."" Policy and Internet. 6(2): 1944-2866.

Earl, Jennifer. 2015. "The Future of Social Movement Organizations: The Waning Dominance of SMOs Online." American Behavioral Scientist 59: 35-52.

Earl, Jennifer, and Katrina Kimport. 2011. Digitally Enabled Social Change: Activism in the Internet Age. Cambridge, MA: MIT Press

Ezekiel, Judith. 2002. Feminism in the Heartland. Columbus, OH: Ohio State University Press.

Fowler, Christopher S., Leif Jensen, and Danielle C. Rhubart. 2019. "Assessing U.S. Labor Market Delineations for Containment, Economic Core, and Wage Correlation." Retrieved November 25, 2018, (https://osf.io/t4hpu/).

Go, Alec, Richa Bhayani, and Lei Huang. 2009. "Twitter Sentiment Classification Using Distant Supervision." CS224N Project Report, Stanford 1: 12.

Graham, Mark, Matathew Zook, and Andrew Boulton. 2012. "Augmented Reality in Urban Places: Contested Content and Duplicity of Code." Transactions of the Institute of British Geographers 38: 464-479.

Grodach, Carl and Renia Ehrenfeucht. 2016. Urban Revitalization: Remaking Cities in a Changing World. New York: Routledge.

Guenther, Katja M. 2010. Making Their Place: Feminism after Socialism in Eastern Germany. Stanford, CA: Stanford University Press.

Hallet, Ronald E. and Kristen Barber. 2014. "Ethnographic Research in a Cyber Era." Journal of Contemporary Ethnography 43.3: 306-330.

Helmuth, Allison Suppan. 2019. "Chocolate City, Rest in Peace: White Space-Claiming and the Exclusion of Black People in Washington, DC." City and Community 18(3): 746-769.

Hinneburg, F. Rosner, M. Roder, M. Nettling, and A. Both. 2014. "Evaluating Topic Coherence Measures." Arxiv:1403.6397 [cs.LG].

Hunter, Marcus Anthony, and Zandria Robinson. 2018. Chocolate Cities: The Black Map of American Life. Oakland, CA: University of California Press.

Hurwitz, Heather McKee, and Verta Taylor. 2012. "Women's Cultures and Social Movements in Global Contexts." Sociology Compass 6(10): 808-822.

Iaquinto, Benjamin L. 2011. "Fear of a Lonely Planet: Author Anxieties and the Mainstreaming of a Guidebook." Current Issues in Tourism 14(8): 705-723.

Janovy, C. J. 2018. No Place Like Home: Lessons in Activism. Lawrence, KS: University Press of Kansas.

Jurafsky, Daniel, and James H. Martin. Forthcoming. Speech and Language Processing, third edition. Accessed February 12, 2019 (https://web.stanford.edu/ jurafsky/slp3/).

Lees, Loretta, Tom Slater, and Elvin Wyly. 2008. Gentrification. New York: Routledge. 
Lim, Kwan Hui, and Amitava Datta. 2012. "Following the Follower: Detecting Communities with Common Interests on Twitter." Proceedings of the 23rd ACM conference on Hypertext and social media 12: 317-318.

Liu, Bing. 2015. Sentiment Analysis: Mining Opinions, Sentiments, and Emotions. New York: Cambridge University Press.

Massey, Douglas, and Nancy A. Denton. 1993. American Apartheid: Segregation and the Making of the Underclass. Cambridge, MA: Harvard University Press.

McCammon, Holly J., Courtney Sanders Muse, Harmony D. Newman, and Teresa M. Terrell. 2007. "Movement Framing and Discursive Opportunity Structures: The Political Success of the U.S. Women's Jury Movements." American Sociological Review 72(5): 725-749.

Molotch. 2002. "Place in Product." International Journal of Urban and Regional Research 26(4): 665-688.

Molotch, Harvey, William Freudenburg, and Krista E. Paulsen. 2000. "History Repeats Itself, But How? City Character, Urban Tradition, and the Accomplishment of Place." American Sociological Review 65(6): 791-823.

Nelson, Laura K. 2017. "Computational Grounded Theory: A Methodological Framework." Sociological Methods \& Research 49(1): 1-40.

Pang, Bo, Lillian Lee, and Shivakumar Vaithyanathan. 2002. "Thumbs up?: Sentiment Classification Using Machine Learning Techniques." Proceedings of the ACL-02 Conference on Empirical Methods in Natural Language Processing 10: 79-86.

Paulsen, Krista. 2004. "Making Character Concrete: Empirical Strategies for Studying Place Distinction." City and Community 3(3): 243-62.

Polletta, Francesca and James M. Jasper. 2001. "Collective Identity and Social Movements." Annual Review of Sociology 27: 283-305.

Princeton University. 2010. "About WordNet." WordNet. Princeton University. Retrieved May 22, 2018 (http://wordnet.princeton.edu).

Quillian, Lincoln. 2012. "Segregation and Poverty Concentration: The Role of Three Segregations.” American Sociological Review 77(3): 354-79.

Reger, Jo. 2012. Everywhere and Nowhere: Contemporary Feminism in the United States. New York: Oxford University Press.

Roder, Michael, Andreas Both, Alexander Hinneburg. 2015. "Exploring the Space of Topic Coherence Measures." Proceedings of the Eighth ACM International Conference on Web Search and Data Mining 2-6: 1-10.

Rohlinger, Deana A., and Jordan Brown. 2009. "Democracy, Action, and the Internet After 9/11." American Behavioral Scientist 53(1):133-50.

Rohlinger, Deana A., Leslie A. Bunnage, and Jesse Klein. 2014. "Virtual Power Plays: Social Movements, Internet Communication Technology, and Political Parties." In Benard Grofman, Alexander H. Treschsel, and Mark Franklin (eds.), The Internet and Democracy in Global Perspective: Voters, Candidates, Parties, and Social Movements, Studies in Public Choice: pp. 83-109. New York: Springer.

Scarborough, William J. 2018. "Feminist Twitter and Gender Attitudes: Opportunities and Limitations to Using Twitter in the Study of Public Opinion." Socius: Sociological Research for a Dynamic World 4: 1-16.

Scarborough, William J., Ray Sin, and Barbara Risman. 2019. "Attitudes and the Stalled Gender Revolution: Egalitarianism, Traditionalism, and Ambivalence from 1977 through 2016." Gender \& Society 33(2):173-200. 
Spain, Daphne. 2017. Constructive Feminism: Women's Spaces and Women's Rights in the American City. Ithaca, NY: Cornell University Press.

Taylor, Keeanga-Yamahtta. 2016. From \#BlackLivesMatter to Black Liberation. Chicago, IL: Haymarket Books.

Turley, Emma, and Jenny Fisher. 2018. "Tweeting Back While Shouting Back: Social Media and Feminist Activism." Feminism \& Psychology 28: 128-132.

Varol, Onur, Emilio Ferrara, Clayton A. Davis, Filippo Menczer, Alessandro Flammini. 2017. "Online Human-Bot Interactions: Detection, Estimation, and Characterization." Proceedings of the Eleventh International AAAI Conference on Web and Social Media (ICWSM 2017) 2017: 280-289.

Wickham, Hadley. 2019. Package 'rvest'. R Software Package (https://cran.rproject.org/web/packages/rvest/rvest.pdf).

Widener, Michael J., and Wenwen Li. 2014. "Using Geolocated Twitter Data to Monitor the Prevalence of Healthy and Unhealthy Food References across the US." Applied Geography 54:189-97. 